\title{
Molecular Profiling of Endometrial Malignancies
}

\author{
Norasate Samarnthai, ${ }^{1}$ Kevin Hall, ${ }^{2}$ and I-Tien Yeh $^{1}$ \\ ${ }^{1}$ Department of Pathology, University of Texas Health Science Center at San Antonio, San Antonio, TX 78229, USA \\ ${ }^{2}$ Department of Obstetrics and Gynecology, University of Texas Health Science Center at San Antonio, San Antonio, TX 78229, USA
}

Correspondence should be addressed to I-Tien Yeh, yehi@uthscsa.edu

Received 1 October 2009; Revised 9 December 2009; Accepted 14 January 2010

Academic Editor: Paul J. Hoskins

Copyright (C) 2010 Norasate Samarnthai et al. This is an open access article distributed under the Creative Commons Attribution License, which permits unrestricted use, distribution, and reproduction in any medium, provided the original work is properly cited.

Molecular profiling of endometrial neoplasms reveals genetic changes in endometrial carcinomas that support the dualistic model, in which type I carcinomas are estrogen-dependent, low grade lesions and type II carcinomas are nonestrogen dependent and high grade. The molecular changes in type I endometrial carcinomas include mutations in PTEN, PIK3CA, KRAS, and $\beta$-catenin, along with microsatellite instability, whereas type II endometrial carcinomas are characterized by genetic alterations in $p 53$, HER2/neu, p16, and E-cadherin. For endometrial neoplasms with a malignant mesenchymal component, C-MYC mutations and loss of heterozygosity are frequently seen in carcinosarcomas, and a fusion gene, JAZF1/JJAZ1, is distinctive for endometrial stromal sarcoma. In addition, $p 53$ mutations may play an important role in tumorigenesis of undifferentiated endometrial sarcoma. These molecular changes can help in the diagnosis of endometrial neoplasms, as well as form the basis of molecular targeted therapy.

\section{Introduction}

Endometrial malignancies can be categorized into two main groups based on the cell of origin: (i) endometrial carcinoma including carcinosarcoma and (ii) endometrial stromal sarcoma. Endometrial carcinomas show a broad spectrum of phenotypes which show various histologic appearances for example, endometrioid, serous, mucinous, squamous, urothelial, or clear cell, reflecting the differentiation potential of the müllerian epithelium and the difference in the tumorigenetic pathways of each tumor type. Women with an inherited predisposition for endometrial neoplasm have been reported, associated with autosomal dominant disorders such as hereditary nonpolyposis colorectal carcinoma (HNPCC) and Cowden syndrome. Some endometrial carcinomas undergo mesenchymal differentiation and are termed carcinosarcomas (formerly termed malignant mixed müllerian tumors). Pathogenetically and clinically, two distinct forms of endometrial adenocarcinoma, type I and type II, have been described. The molecular alterations driving endometrial carcinogenesis may follow a sequence similar to Vogelstein's model for the progression of colorectal adenoma to carcinoma. This process is accompanied by stepwise genetic changes of oncogenes and tumor suppressor genes. Endometrial stroma may give rise to neoplasms that resemble normal endometrial stromal cells. The spectrum of endometrial stromal tumors ranges from the benign stromal nodule to the malignant endometrial stromal sarcoma. An oncogenic fusion gene, JAZF1/JJAZ1 plays a significant role in tumor development of endometrial stromal sarcomas [1].

\section{Molecular Profiling of Endometrial Carcinoma}

2.1. Dualistic Model of Endometrial Tumorigenesis. Endometrial carcinoma is the most common malignant neoplasm of female genital tract in developed countries [2] with an estimated 42,160 new cases diagnosed in the United States for 2009 [3]. Approximately 90\% of cases of endometrial carcinoma are sporadic, whereas the remaining $10 \%$ of cases are hereditary [4]. Clinically, the patients with endometrial carcinomas most often present with abnormal uterine bleeding. In advanced stages, patients may complain of pelvic pain, reflecting spread of the carcinoma. Bokhman [5] first described the pathogenetic classification of 2 different types of endometrial carcinoma, designated as type I and type II carcinomas, according to the determination of biological properties of the tumor, its clinical course, and the prognosis of the disease. 


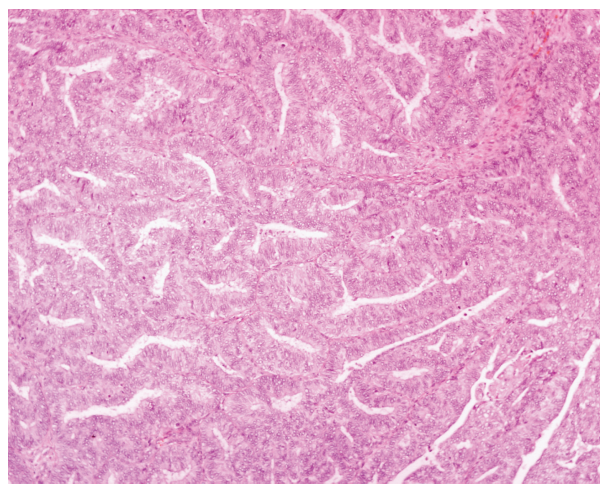

(a)

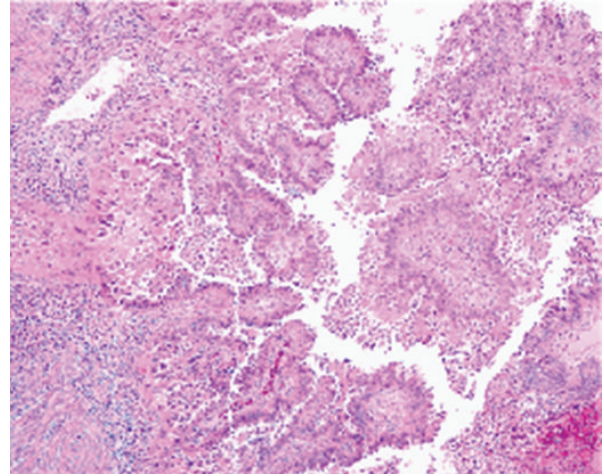

(b)

FIgURE 1: The prototypes for the dualistic model of endometrial carcinoma. Type I endometrioid endometrial carcinoma shows glands lined by stratified neoplastic columnar cells (a), $\times 100$; and type II serous carcinoma showing papillary structures and high nuclear grade (b), $\times 100$.

2.1.1. Type I (Endometrioid Endometrial Carcinoma) [1, 2, 413]. Type I carcinomas represent the majority of sporadic cases of endometrial carcinoma, accounting for $70-80 \%$ of new cases [4, 9-12] which occur predominantly in preand perimenopausal women. These cancers are typically of endometrioid type (Figure 1(a)). Risk factors include obesity, hyperlipidemia, and hyperestrogenism for example, anovulation, nulliparity/infertility, late onset of menopause, and endometrial hyperplasia. The tumors in this category are generally low-grade, low-stage, and indolent. They commonly express estrogen and progesterone receptors [2, 4-11]. The rare mucinous carcinomas are also considered type I carcinomas because they usually express estrogen and/or progesterone receptors and are of low histologic grade $[10,11]$.

2.1.2. Type II (Nonendometrioid Endometrial Carcinoma) [1, 2, 4-13]. Type II carcinomas are less common, accounting for $10-20 \%$ of endometrial carcinoma $[4,6]$. They are nonendometrioid in differentiation, most frequently papillary serous (Figure 1(b)) and less frequently clear cell, have high-grade histology, typically arise in an atrophic endometrial background, and often have deep myometrial penetration. They usually occur at an older age, approximately 510 years later than type I tumors. There is no relationship to estrogen stimulation. Clinically, type II cancers have an aggressive behavior, with a high frequency of distant spread to pelvic lymph nodes. Small cell, undifferentiated and squamous cell carcinomas may also be encountered among type II carcinomas, but little is known about their tumorigenesis [11]. The clinical and pathological features of the two types of endometrial carcinomas are summarized in Table 1 .

2.2. Common Molecular Genetic Alterations in Dualistic Model. Evidence for divergent molecular alterations supporting the dualistic model of endometrial tumorigenesis became available approximately 10 years after Bokhman's description of the clinical and pathologic features. The two distinct histological types of carcinomas are associated with genetic alterations of independent sets of genes. These genetic changes may occur singly or in various combinations which differ between individual cases [9].

Westin and colleague [14] described that expression of estrogen-induced genes, RALDH2, EIG121, SFRP1, SFRP4, $I G F-1$, and $I G F-I R$, tend to be highest in the well-tomoderately differentiated endometrioid carcinoma. This finding supports the partitioning of endometrial carcinoma into two distinct groups by traditional estrogen-related classification. According to this model, normal endometrial cells would transform into endometrioid endometrial carcinoma through 5 different molecular changes, including, mutations of PTEN, PIK3CA, KRAS, and CTNNB1 $(\beta-$ catenin) genes and microsatellite instability (MSI) while non-endometrioid endometrial carcinoma is frequently related to alterations of $p 53$ and chromosomal instability $[7,8,15,16]$. Non-endometrioid endometrial carcinoma frequently demonstrates high-ordered aneuploidy and has an intact mismatch repair (MMR) mechanism [12]. Furthermore, none of the five main alterations of endometrioid endometrial carcinoma (mutations of PTEN, PIK3CA, $K R A S$, and CTNNB1 genes and MSI) plays a major role in non-endometrioid endometrial carcinoma. However, in many endometrial carcinomas exhibit overlapping clinical, morphologic, immunohistochemical, and molecular features of the both types of carcinoma for example, a subset of endometrioid endometrial carcinoma is found with a background of atrophic endometrium or papillary serous carcinoma may occasionally develop from a pre-existing endometrioid endometrial carcinoma and may share histological and genetic features [8-10]. Matias-guiu et al. [8] described the development of non-endometrioid endometrial carcinoma through these possible pathways: (i) de novo, through p53 mutations, loss of heterozygosity (LOH) at several loci, and some other still unknown gene alterations; or (ii) through dedifferentiation of a pre-existing endometrioid carcinoma. These dedifferentiated non-endometrioid endometrial carcinomas exhibit overlapping features with type I endometrioid endometrial carcinoma [8]. 
TABLE 1: Clinical and pathological characteristics of type I and type II endometrial carcinoma [1, 2, 4-13].

\begin{tabular}{lcc}
\hline & Type I & Type II \\
\hline Proportion of endometrial carcinomas & $4 / 5$ & $1 / 5$ \\
Menstrual status & Pre- and perimenopausal & Postmenopausal \\
Endocrine-metabolic disturbance & Present & Absent \\
Estrogen-associated & Hyperplasia & No \\
Background endometrium & Endometrioid & Atrophy \\
Histological type & Low & Serous, clear cell \\
Tumor grade & Superficial & High \\
Depth of myometrial invasion & Stable/indolent & Deep \\
Behavior & & Progressive/aggressive \\
\hline
\end{tabular}

Comparison of the major genetic alterations between type I and type II endometrial carcinomas is shown in Table 2.

Molecular genetic alterations have been extensively investigated in endometrioid and papillary serous adenocarcinomas of the endometrium. These two tumor types are characterized by distinctive molecular alterations, and their tumorigenesis follow separate pathways.

\subsection{Molecular Pathology of Endometrioid Carcinomas}

2.3.1. PTEN. The most frequently altered gene in endometrioid endometrial carcinoma is PTEN (phosphatase and tensin homologue deleted from chromosome 10), also called MMAC1 (mutated in multiple advanced cancers 1). PTEN behaves as a tumor suppressor gene, is located on chromosome 10q23.3 and encodes a lipid phosphatase that antagonizes the PI3K/AKT pathway by dephosphorylating PIP3, the product of PI3K. This lipid molecule is an important second messenger that regulates the phosphorylation of a protein termed AKT, also known as protein kinase B. Decreased PTEN activity causes increased cell proliferation and survival through modulation of signal transduction pathways.

PTEN may be inactivated by several mechanisms such as mutation, $\mathrm{LOH}$, and promoter hypermethylation. Somatic PTEN mutations are common in endometrial carcinoma, and they are almost exclusively restricted to endometrioid endometrial carcinomas, occurring up to $83 \%$ of them [1, $4,11,12]$. Germline mutations of PTEN are responsible for Cowden syndrome $[9,12]$. PTEN may be also inactivated by deletion, as shown by $\mathrm{LOH}$ in $40 \%$ of endometrial carcinomas $[7-9,17]$. Promoter hypermethylation leading to PTEN inactivation, is found in about $20 \%$ of tumors, most of which are high-stage [10].

PTEN mutations have been detected in $15-55 \%$ of endometrial hyperplasias with and without atypia $[9,13]$. Interestingly, concordance between MSI status and PTEN mutations has been found; the mutations occur in $60-86 \%$ of MSI-positive endometrioid endometrial carcinoma but in only $24-35 \%$ of the MSI-negative cases [7-9, 13, 17]. This suggests that PTEN could be a target for mutations in the context of DNA repair deficiency [13]. In addition, identical PTEN mutations have been also identified in hyperplasias coexisting with MSI-positive endometrioid endometrial carcinoma, which suggests that PTEN mutations are early events in their development [8]. On the other hand, identical PTEN mutations have been detected in MSI-negative endometrial hyperplasia with coexisting MSIpositive endometrioid endometrial carcinomas. Thus, some PTEN mutations may precede MSI, and coexistence of both alterations does not necessarily mean a cause-effect relationship [9]. Evaluation of PTEN inactivation in endometrial carcinoma precursor lesions by PTEN immunostaining has been proposed. However, commercially available antibodies (e.g., clone 10P03, 28H6, polyclonal, 6H2.1) do not have statistically significant associations with the molecular genetic alterations $[7,9,19]$. Some data suggest that PTEN is associated with younger age, low stage, endometrioid histology, low histologic grade, and favorable prognosis (78\% 5 -year survival for patients without mutations, compared with $95 \%$ and $93 \%$ for patients with one or more mutations, resp.) $[7,9]$. In addition, recent data suggest that only PTEN mutations outside exons 5-7 may predict favorable survival, independent of the clinical and pathological features of the tumors [9].

2.3.2. PIK3CA. The PIK3CA (p110 $\alpha$ catalytic subunit of PI3K) gene locates on chromosome 3q26.32. Phosphatidylinositol-3-kinase (PI3K) is heterodimeric lipid kinase consisting of a catalytic subunit (p110) and a regulatory subunit (p85) in PI3K/AKT signaling pathway. This pathway is frequently activated in endometrial carcinoma through various genetic alterations and their combinations. Activation of PI3K produces the second messenger PIP3 which subsequently activates various down-stream pathways such as AKT. This regulation involves suppression of apoptosis and enhancement of cell proliferation [9]. PIK3CA activation is reported in $26-36 \%$ of endometrial carcinoma and may coexist with PTEN (15-27\%) $[7,9,12,15,20]$ and KRAS mutations $[9,15,20]$ suggesting that the PIK3CA mutations cooperate with these alterations in malignant transformation [16]. Mutations in $A K T$ family members and their correlation with other gene alterations are found in endometrial carcinoma, 
TABLE 2: Genetic alterations of type I and type II endometrial carcinomas, reported in percentages (references).

\begin{tabular}{lcc}
\hline & Type I & Type II \\
\hline PTEN inactivation & Up to $83 \%[1,4,11,12]$ & $11 \%[1,2,12]$ \\
PIK3CA mutation & $26-36 \%[7,9]$ & $5 \%[7]$ \\
KRAS mutation & $10-30 \%[1,2,4,7-12,17]$ & $0-10 \%[2,12]$ \\
$\beta$-catenin /CTNNB1 mutation & $14-44 \%[7,8]$ & $0-5 \%[1,7,10,11]$ \\
Microsatellite instability & $20-45 \%[1,7-10]$ & $0-11 \%[8,9]$ \\
p53 mutation & $10-20 \%[1,4,6,7,10,11,13,17,18]$ & $90 \%[1,2,4,6,7,10-13,17]$ \\
HER2/neu amplification & $10-30 \%[1,4,10,17]$ & $18-80 \%[13]$ \\
p16 inactivation & $10 \%[1,4,7,10,11]$ & $40-45 \%[4,7,10]$ \\
E-cadherin loss & $10-20 \%[1,4,7,10,11]$ & $60-90 \%[4]$ \\
\hline
\end{tabular}

including $A K T 2(\mathrm{D} 399 \mathrm{~N}), A K T 2(\mathrm{D} 32 \mathrm{H})$ and $A K T 3$ (E438D) mutations. Mutations of AKT3 (E438D) also have amplification of and a mutation in PIK3CA [21]. AKT1 E17K mutation is not associated with either PTEN or PIK3CA genomic alteration [21]. In vitro studies showed that activating mutations of PIK3CA in combination with PTEN mutations led to an additional increase in phosphorylated AKT when compared with cells with only inactivated PTEN [6]. Some investigators have claimed that PIK3CA mutations are mutually exclusive of PTEN mutations, suggesting that tumorigenic signaling through this pathway can occur either through activation of PIK3CA or inactivation of PTEN [9]. Recently, interactions between the PI3K/AKT and $p 53$ signaling pathways have been described in which activation of the PI3K/AKT pathway through PTEN or PIK3CA mutations, together with $p 53$ inactivation, results in malignant transformation [15]. Moreover, patients with dysregulation of PI3K/AKT signaling pathway and p53 alterations had shorter survival than patients with only p53 alterations [15]. Mutations were more common in mixed endometrioid-nonendometrioid adenocarcinomas $(44 \%)$ than in pure endometrioid adenocarcinomas $(28 \%)$ or pure nonendometrioid adenocarcinomas (21\%) [15]. In fact, PIK3CA mutations are usually missense and cluster in exons 9 (helical domain) and 20 (kinase domain). The tumors carrying exon 9 PIK3CA mutations are more likely to be low-grade carcinomas; in contrast, carcinomas with exon 20 mutations or PIK3CA mRNA overexpression are often high-grade carcinomas associated with myometrial invasion and tended to have lymphovascular invasion [15]. Furthermore, in high-grade endometrioid adenocarcinomas and mixed carcinomas, PIK3CA mutations in exon 20 coexist with $p 53$ alterations more frequently than in nonendometrioid adenocarcinomas. However, PIK3CA mRNA overexpression occurs in concert with $p 53$ alterations only in nonendometrioid endometrial carcinomas [15]. PIK3CA mutations did not correlate with MSI or $\beta$-catenin/CTNNB1 mutations $[9,18]$. PIK3CA mutations, particularly exon 20 mutations or PIK3CA mRNA overexpression, are frequent in endometrioid endometrial carcinoma in association with invasion and adverse prognostic factors such as blood vessel invasion $[7,15]$.
2.3.3. KRAS. KRAS encodes a member protein of the small GTPase superfamily and is involved in signal transduction pathways between cell surface receptors and the nucleus. KRAS mutations have been identified in 10-30\% of endometrioid endometrial carcinomas [1, 2, 4, 7-12, 17] while some investigators have reported an almost complete absence of KRAS mutations in serous and clear cell carcinomas of endometrium [8]. Some studies found a higher frequency of KRAS mutations in MSI-positive carcinomas than in MSI-negative tumors [8-10, 16] suggesting that both events may occur simultaneously before clonal expansion $[10,13]$. KRAS mutations were detected in endometrial hyperplasias at a similar rate to that observed in endometrioid endometrial carcinomas, suggesting that KRAS mutations are early events in endometrial carcinogensis $[9,13]$. No relationship has been found between KRAS mutations and tumor stage, histologic grade, depth of myometrial invasion, age, or clinical outcome in endometrioid endometrial carcinomas [9].

2.3.4. $\beta$-Catenin (CTNNB1). The $\beta$-catenin gene (CTNNB1) maps to $3 \mathrm{p} 21$. It appears to be important in the functional activities of both APC (adenomatous polyposis coli) and E-cadherin. It is a component of the E-cadherin-catenin unit, essential for cell differentiation and maintenance of normal tissue architecture and also plays an important role in Wnt signal transduction pathway. Mutations in exon 3 of CTNNB1 result in stabilization of a protein that resists degradation, leading to nuclear accumulation of $\beta$-catenin, have been described in endometrioid endometrial carcinoma. The accumulation of $\beta$-catenin can be demonstrated by immunostaining. Several studies have analyzed endometrial carcinomas showing that nuclear accumulation of $\beta$-catenin is significantly more common in endometrioid lesions (31$47 \%)$ compared with nonendometrioid histology (0-3\%) [4]. By comparison in colonic adenocarcinomas, elevated $\beta$ catenin levels caused by mutations in CTNNB1 or APC result in activation of the $\mathrm{Wnt} / \beta$-catenin/LEF1 pathway through a LEF1 binding site in the cyclin D1 promotor, triggering cyclin D1 gene expression, and subsequently, uncontrolled progression of tumor cells into the cell cycle $[8,12]$. Furthermore, $\beta$-catenin might regulate the expression of 
the matrix metalloproteinase- 7 that would have a role in the establishment of the microenvironment necessary for the initiation and maintenance of growth of the primary tumor and metastasis $[8,12]$. The reported frequency of CTNNB1 mutations in endometrioid endometrial carcinoma ranges from $14-44 \%[7,8]$. They seem to be independent from the presence of MSI and the mutations of PTEN and KRAS, suggesting that the Wnt pathway may play an independent role in endometrial cancer $[10,13]$. In all cases, the mutations were homogeneously distributed in different areas of the tumors suggesting that they play a role in early steps of endometrial tumorigenesis. Alterations in $\beta$ catenin have been reported in endometrial hyperplasias with squamous metaplasia $[7,9]$. Although there was a good correlation between CTNNB1 mutations and $\beta$-catenin nuclear immunostaining, the presence of cytoplasmic and nuclear $\beta$ catenin immunoreactivity in some endometrial carcinomas without $C T N N B$ mutation suggests that the changes of other genes in the $\mathrm{Wnt} / \beta$-catenin/LEF-1 pathway may be responsible for the stabilization and putative transcription activator role of $\beta$-catenin $[7,8]$. Endometrioid endometrial carcinomas with CTNNB1 mutations are characteristically early stage tumors associated with favorable prognosis $[7,9]$. Two members of the secreted frizzled-related protein (SFRP) family, SFRP1 and SFRP4, were more frequently downregulated in MSI-positive carcinomas compared with MSInegative carcinomas. This down-regulation was associated with frequent promoter methylation of SFRP1 and led to an activation of the $\beta$-catenin pathway. In addition, the Wnt-target fibroblast growth factor 18 was up-regulated in endometrioid carcinomas with MSI compared with normal endometrium [1].

\subsubsection{Microsatellite Instability. Microsatellite DNA sequen-} ces are polymorphic, short-tandem repeats distributed throughout the genome. The most common microsatellite in human is a dinucleotide repeat of CA, (CA)n, and there are 50,000 to $100,000(\mathrm{CA}) \mathrm{n}$ repeats scattered in the human genome $[8,9]$. Microsatellite instability (MSI) is a condition manifested by damaged DNA because of defects in normal DNA repair process. Mammalian mismatch repair (MMR) genes encode for nine proteins (MLH1, MLH3, PMS1, PMS2, MSH2, MSH3, MSH4, MSH5, and MSH6) that interact with each other to form complexes and heterodimers that mediate distinct functions in MMR-related system. This repair process plays a central role in promoting genetic stability by repairing DNA replication errors, inhibiting recombination between non-identical DNA sequences and participating in responses to DNA damage. MSI is a common genetic abnormality that has been detected in $20-45 \%$ of sporadic endometrioid endometrial carcinoma [7-10]. In addition, MSI in nonendometrioid endometrial carcinomas has been reported $(0-11 \%)[8,9]$, particularly in mixed endometrioid and serous carcinomas, but not in pure serous carcinomas [10]. In sporadic endometrial carcinoma, epigenetic cause of MSI is more common involving MLH1 promotor hypermethylation which is the main cause of MMR deficiency $[7-9,13,15]$. This epigenetic inactivation usually occurs in atypical hyperplasia, most of which coexists with carcinomas. Thus, MLH1 hypermethylation is an early event in the pathogenesis of endometrioid endometrial carcinoma, which precedes the development of MSI [7-9, 15]. The remaining unmethylated MLH1 cases reveal MSH2 mutations (15\%) and MSH6 mutations (60\%), of which almost half are germline mutations. Thus, MSH6 mutations seem to be a frequent cause of MSI $[11,12]$. Tumors with MSI of CpG island methylation in the promoter region have been identified in some other genes, for example, p16, PTEN, and E-cadherin $(\mathrm{CDH} 1)$, suggesting altered methylation may be a coexisting independent early change [9]. The presentation of some small short-tandem repeats such as mononucleotide repeats located within the coding sequence of important genes for example, transforming growth factor $\beta$ receptor type II (TGF- $\beta R I I), B A X$, insulin-like growth factor II receptor (IGFIIR), MSH3, MSH6, caspase-5, and PTEN may promote MSI-positive endometrial carcinoma $[8,9]$. Secondary mutations at one or more mononucleotide tracts found in $72.7 \%$ of tumors with MSI, are responsible for tumor progression [7-9]. International Federation of Gynecology and Obstetrics (FIGO) grade has been found to be higher in endometrioid endometrial carcinomas with MSI in some, but not all studies, similar to the well-established association between MSI and high-grade colorectal carcinomas [16]. By multivariate analysis, a significant correlation between MSI-positive tumors and tumor-infiltrating lymphocytes in endometrioid endometrial carcinoma was found: 40 tumor-infiltrating lymphocytes/10 high power fields has a sensitivity of $85 \%$ and a specificity of $46 \%$ in predicting MSI [16].

\subsection{Molecular Pathology of Nonendometrioid Carcinomas}

2.4.1. $p 53$. The $p 53$ tumor suppressor gene locates to chromosome $17 \mathrm{p} 13.1$. While p53 mutations occur in $90 \%$ of non-endometrioid endometrial carcinoma, they are only present in $10-20 \%$ of endometrioid endometrial carcinoma, which are mostly high-grade $[7,18]$. The abnormal p53 expression has been found in $11 \%$ of grade 1 endometrioid endometrial carcinoma [18]. This finding supports that p53 mutations may influence progression of endometrioid endometrial carcinomas to non-endometrioid endometrial carcinomas [9]. In fact, p53 mutation is the most characteristic genetic alteration of non-endometrioid endometrial carcinomas $[9,10]$ and may be useful in their distinction from endometrioid endometrial carcinomas [22]. In p53 positive endometrioid endometrial carcinoma, p53 protein accumulation may be secondary to changes in its upstream regulatory proteins rather than the $p 53$ gene itself. Several genes, including $M D M 2$ and $p 14 A R$, that regulate $p 53$ levels have been shown to cause detectable levels of $p 53$ in the absence of p53 mutation. Alternatively, nonspecific DNA damage such as that induced by irradiation is also known to induce accumulation of wild-type p53 [12]. In normal cells, $p 53$ is rapidly degraded and thus cannot be detected by immunostaining. $p 53$ mutations produce a non-functional protein that resists degradation and can be visualized by 
immunostaining $[11,18]$. However, loss of function of p53 resulting from LOH may not correlate with protein overexpression. In addition, frameshift mutations and stop codons lead to a truncated protein, which is not detected by antibodies and leads to negative immunohistochemistry [11, 18] After DNA damage, nuclear $p 53$ accumulates and causes cell cycle arrest by inhibiting cyclin D1 phosphorylation of the $R b$ gene and thereby promoting apoptosis $[9,13]$. Overexpression of $p 53$ is associated with high histological grade and advanced stage as well as unfavorable prognosis $[9,18]$. Endometrial intraepithelial carcinoma (EIC), the putative precursor lesion to serous carcinomas $[4,13,18,22$, 23], characterized by replacement of the surface epithelium by malignant cells exhibiting cytological features similar to those of serous carcinoma $[9,23]$. EIC has been reported in nearly $90 \%$ of uteri containing serous carcinoma that is often extensive and multifocal [23]. Mutations of $p 53$ are also found in $75-80 \%$ of EIC. It is postulated that mutation in one allele occurs early during the development of serous carcinoma, and loss of the second normal allele occurs late in the progression to carcinoma [4]. $p 53$ mutations are almost always associated with aneuploidy and do not seem to occur with PTEN mutations in the same tumor $[10,11]$.

2.4.2. HER2/neu. Epidermal growth factor receptor II or HER2/neu is an oncogene that codes for a transmembrane receptor tyrosine kinase involved in cell signaling and located at the long arm of human chromosome 17q12. HER2/neu overexpression or amplification is more frequently found in non-endometrioid endometrial carcinoma (18-80\%) [13] than in grade 2 and 3 endometrioid carcinoma (10-30\%) $[7,9,10]$ and has been associated with adverse prognostic parameters including advanced stage, high histologic grade, and low overall survival $[9,13]$.

2.4.3. $p 16 . p 16$ plays an important role in regulating the cell cycle. It is a tumor suppressor gene located on chromosome 9 p21 [10]. p16 inactivation can lead to uncontrolled cell growth. Inactivation of $p 16$ is more frequent in nonendometrioid endometrial carcinoma $(40-45 \%)$ than in endometrioid endometrial carcinoma $(10 \%)[4,7,10]$. The underlying mechanism is unclear $[7,11]$, because neither promoter hypermethylation nor deletion or mutation is frequently found [11]. Loss of p16 expression is correlated with KRAS and p53 mutations and is associated with high stage, high grade, and poor survival [10].

2.4.4. E-Cadherin. Cadherins are a family of adhesion molecules essential for tight connection between cells. Ecadherin is encoded by $\mathrm{CDH} 1$ gene and locates on chromosome 16q22.1. It is thought to be a tumor suppressor gene, the loss of which has been demonstrated to promote tumor invasion and metastasis. Decreased expression of E-cadherin is frequent in endometrial carcinoma and may be caused by $\mathrm{LOH}$ or promotor hypermethylation. $\mathrm{LOH}$ at $16 \mathrm{q} 22.1$ is seen in almost $60 \%$ of non-endometrioid endometrial carcinoma, but in only $22 \%$ of endometrioid endometrial carcinoma
[7]. In endometrial carcinoma, partial or complete loss of Ecadherin expression correlates with aggressive behavior [9].

Among type II carcinomas, clear cell carcinomas seem to follow a separate pathway that shows some overlap with serous and endometrioid carcinomas. $p 53$ mutations are only present in about $30-40 \%$ of clear cell carcinomas compared to $90 \%$ of serous carcinomas. However, the frequency of MSI and PTEN alterations in clear cell carcinoma is higher than in serous carcinoma ( $15 \%$ versus $<5$ for MSI and $30 \%$ versus $10 \%$ for PTEN) but lower compared with endometrioid carcinoma (20-40\% and 35-50\%, resp.) [24]. A recent molecular study demonstrated that the majority of pure clear cell carcinomas do not show mutations in either PTEN or $p 53$, the most commonly altered genes in type I and type II tumors, respectively. These findings suggest that clear cell carcinoma may arise through a distinct pathologic pathway [6].

2.4.5. Apoptosis Resistance in Endometrial Carcinoma. Several of the molecular abnormalities that have been detected in EC may be associated with apoptosis deregulation. Apoptosis can be initiated by two main mechanisms: (i) the "intrinsic pathway" activated by released mitochondrial proteins, such as cytochrome-c; and (ii) the "extrinsic pathway" activated by ligand-bound death receptors such as tumor necrosis factor (TNF), Fas or TNF-related apoptosis including ligand (TRAIL) receptors. Some studies have shown that cellular apoptosis susceptibility $(C A S)$ gene, $B C L 2, B A X$, and caspase-3 are apparently involved in the progressive deregulation of proliferation and apoptosis, leading from simple and complex endometrial hyperplasia to adenocarcinoma. As described above, PTEN antagonizes the PI3K/AKT pathway by dephosphorylating PIP3, resulting in decreased translocation of AKT activation. Thus, loss of PTEN function leads to increased levels of phosphoAKT, activation of anti-apoptotic protein, and cell cycle progression [9]. NF- $\kappa \mathrm{B}$, frequently activated in endometrioid endometrial carcinomas, may inhibit apoptosis by activation of target genes such as FLIP and Bcl-XL. Furthermore, there are reports that apoptosis-related protein survivin is frequently overexpressed in endometrial carcinomas [7, 9] and correlates inversely with PTEN expression [9]. Where widespread genetic abnormalities exist that cannot be corrected, MMR proteins initiate apoptosis as a more energy efficient option of universal genomic preservation [16]. MMR deficiency lowers the apoptotic rate, leading a survival advantage to the mutated cells [16].

2.4.6. cDNA Array Studies. cDNA analyses have demonstrated that the expression profiling of endometrioid endometrial carcinoma is different from that of nonendometrioid endometrial carcinoma. These studies have identified gene signatures specific for non-endometrioid endometrial carcinomas as well as genes specifically upor down-regulated in endometrioid endometrial carcinomas when compared with normal endometrium. Intestinal trefoil protein, TFF3, AGR2 developmental gene, estrogenregulated genes (MGB2, LTF, END1, MMP11), FOXA2, 
and MSX2 were significantly up-regulated in endometrioid endometrial carcinomas, while increased expression of $F O L R$, genes involved in the regulation of mitotic spindle checkpoint (STK15, BUB1, CCNB2), IGF2, PTGS1 and p16 were seen in non-endometrioid endometrial carcinomas. STK-15 also known as BTAK, Aurora-A, is a serine/threonine kinase which is essential for chromosome segregation and centrosome functions [7,9]. Overexpression of STK15 induces increased numbers of centrosomes, aneuploidy, and malignant transformation. One study found STK15 amplification in 9 of $15(60 \%)$ non-endometrioid endometrial carcinomas but in none of endometrioid endometrial carcinomas [9]. Furthermore, a different expression profile was also found between endometrial carcinoma associated with MSI and stable endometrial carcinoma. SFRP1 and SFRP4 were more frequently down-regulated in endometrial carcinoma with MSI. One study compared the expression profiles of similar histological subtypes of ovarian and endometrial carcinomas, and showed that clear cell carcinomas had a very similar profile, regardless of the organ of origin. In contrast, differences were seen when comparing endometrioid and serous carcinomas of ovarian and endometrial origin [7].

\section{Genetic Changes in Endometrial Carcinogenesis (Progression Models) of Endometrioid and Serous Carcinomas, Including Molecular Changes of Premalignant Disease (Hyperplasia/EIC)}

By epidemiological and molecular evidence, endometrial hyperplasia represents a true precursor lesion for endometrioid endometrial carcinomas, whereas non-endometrioid endometrial carcinomas are frequently associated with endometrial intraepithelial carcinoma (EIC) [13].

\subsection{Progression Model for Endometrioid (Type I) Carcinomas.} A progression model of endometrioid carcinoma resembling the Vogelstein progression model for colorectal carcinoma has been proposed. This hypothesis is supported by the evidence that (i) some of the genetic alterations found in endometrioid endometrial carcinomas are already present in atypical hyperplasia, (ii) increased genetic alterations are found in well-differentiated endometrioid carcinoma compared with atypical hyperplasia, (iii) the number of genetic alterations increase according to higher histologic grade, and (iv) more chromosomal imbalances are identified in endometrial carcinoma compared with atypical hyperplasia, using comparative genomic hybridization (CGH) [11].

Most simple hyperplasias and a subset of complex hyperplasias are polyclonal and considered reactive processes due to hyperestrogenism, which may regress through progesterone therapy $[11,23]$. In contrast, most atypical hyperplasias are monoclonal. A subset of complex hyperplasia without atypia has been reported to be monoclonal. In addition, the number of chromosomal aberrations in complex hyperplasia is significantly higher than simple hyperplasia and close to the number found in atypical hyperplasia. Most of the genetic alterations identified in endometrioid

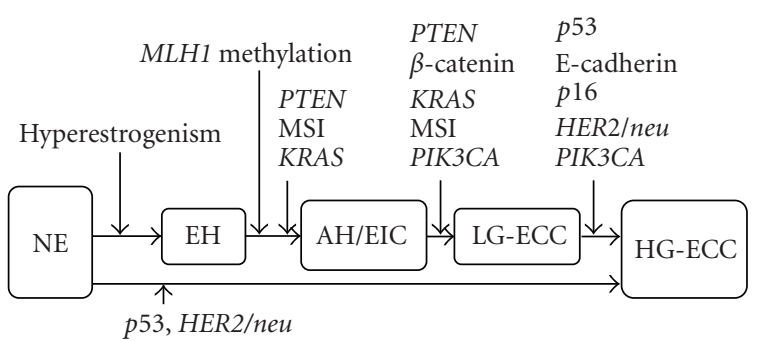

Figure 2: A progression model for endometrioid carcinoma. Tumor initiation and progression are characterized by acquisition of various molecular alterations. PTEN alterations appear central to the initiation of proliferative lesions that then acquire mutations in other cancer-causing genes (e.g., DNA mismatch repair genes, KRAS, $\beta$-catenin) in the carcinogensis. An alternative pathway bypasses atypical hyperplasia and low-grade carcinoma to highgrade carcinoma by $p 53$ mutation and HER2/neu amplification. $\mathrm{NE}$, normal endometrium; $\mathrm{EH}$, endometrial hyperplasia without hyperplasia, AH, atypical endometrial hyperplasia; EIC, endometrial intraepithelial carcinoma; LG-ECC, low grade endometrioid endometrial carcinoma; HG-ECC, high grade endometrioid endometrial carcinoma.

endometrial carcinoma seem to occur very early in the development of endometrioid carcinoma, although it is not clear which alterations are associated with the earliest changes of malignant transformation and progression to neoplasia $[10,11]$. In atypical hyperplasia, alterations of PTEN, $\beta$-catenin, KRAS, and MSI are present, with PTEN inactivation occuring in about $50 \%$ of the cases. However, PTEN and KRAS mutations seem to occur earlier, since they were found in simple hyperplasia, partially associated with monoclonality. PTEN inactivation has been reported in normal endometrial glands but its significance is yet unknown [11]. The inactivation of E-cadherin gene by methylation seems to play a role during progression of endometrioid carcinoma, since it is most frequently found in grade 3 and least frequently in grade 1 tumors [10]. Furthermore, p53 mutations, HER2/neu overexpression or amplification, and p16 inactivation are considered in late events during carcinogenesis of endometrioid carcinoma, since they are predominantly identified in grade 3 tumors, but rarely in grade 1 tumors, and are absent in atypical hyperplastic lesions. Hypothetically, p53 mutations and HER2/neu amplification might also be early events in de novo poorly differentiated endometrioid carcinomas [10, 11] (Figure 2). Endometrial pre-cancers (e.g., EIC) have been postulated to share common genetic alterations with endometrioid endometrial carcinoma, including PTEN mutations and MSI [13].

3.2. Progression Model for Nonendometrioid (Type II) Carcinomas. Mutations of $p 53$ were found in approximately $80 \%$ of EIC, but in contrast to most serous carcinomas, there is no LOH at the locus TP53. Thus, it is hypothesized that p53 mutation of one allele occurs early, whereas loss of the normal second allele accompanies progression into serous carcinoma $[10,11]$. The alterations of E-cadherin, 


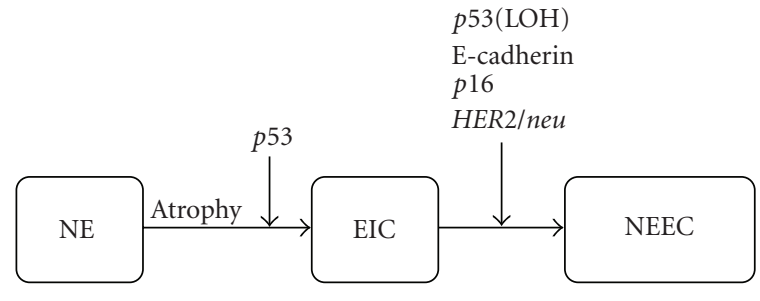

Figure 3: A progression model for nonendometrioid (type II) carcinomas. p53 mutations play a critical role in the conversion of atrophic endometrium to an intraepithelial form of serous carcinoma. NE, normal endometrium; EIC, endometrial intraepithelial carcinoma; NEEC, non-endometrioid endometrial carcinoma.

p16, and HER2/neu seem to affect the progression from EIC to serous carcinoma [10]. Another group hypothesized that serous carcinoma may develop from endometrioid carcinoma through p53 mutation based on findings in mixed endometrioid and serous carcinomas. Early genetic alterations during carcinogenesis are not clear, as these authors presented no data for EIC [10, 11] (Figure 3).

\section{Hereditary Endometrial Carcinoma}

Hereditary endometrial carcinoma has been found in 2-5\% of endometrial cancer [24]. Hereditary nonpolyposis colon cancer (HNPCC), also known as Lynch syndrome or cancer family syndrome, accounts for the majority of inherited cases [24]. It is an autosomal dominant syndrome that predisposes its carriers to multiple malignancies particularly colorectal, and endometrial carcinomas [25], caused by a germline mutation in one of the DNA MMR genes occurring in 30$60 \%$ of cases $[8,9]$. Endometrial carcinoma is the most common extracolonic malignancy in patients with HNPCC. In women with HNPCC, the incidence of endometrial carcinoma equals or exceeds that of colorectal carcinoma, compared with $1 \%$ in the general population [26], and in more than $50 \%$ of HNPCC cases, these women present with a gynecological cancer as their first or "sentinel" malignancy [25]. The frequency of germline DNA MMR gene mutations among unselected patients with endometrial carcinoma has been found to be $1.8-2.1 \%$, which is similar to the frequency of HNPCC in colorectal carcinoma [25]. Patients with endometrial carcinoma in the HNPCC population have an inherited germline mutation in MLH1, MSH2, MSH6, or PMS2 (first hit) but endometrial carcinoma develops only after the initiation of a deletion or mutation in the contralateral MLH1, MSH2, MSH6, or PMS2 allele (second hit) in endometrial cells $[7,8]$. Once the 2 hits have occurred, the deficient MMR function of MLH1, MSH2, MSH6, or PMS2 causes the acquisition of MSI and subsequent tumor development [7-9]. Unlike HNPCC associated colorectal carcinoma, which appears to frequently have $M L H 1$ and MSH2 mutations, endometrial carcinomas have a higher probability of $M S H 2$ and MSH6 mutations [13, 25]. Women with HNPCC who carry MSH2 and MSH6 mutations have a higher chance to present initially with endometrial rather than colorectal cancer [16]. MSI has been detected in $75 \%$ of endometrial carcinoma associated with $\operatorname{HNPCC}[8,9]$. Many studies have shown that MSI is associated with endometrioid histologic type. However, $14-21 \%$ of HNPCCassociated endometrial carcinomas are non-endometrioid, but only 3.3-4.5\% of sporadic MSI tumor [16]. Women with an inherited predisposition for endometrial neoplasia tend to develop the disease 10 years earlier than the general population [9]. There is $18-23 \%$ incidence of HNPCC syndrome in endometrial carcinoma patients younger than 50 years old [16]. In addition to endometrial carcinoma arising from HNPCC, occasional families show clustering of endometrial cancer alone, without colon or other cancers. This group was termed as "familial site-specific endometrial cancer" [10]. Loss of protein expression seems to occur frequently for both $M L H 1$ and $M S H 2$ in endometrial hyperplasia and is considered an early event during tumor development [11]. PTEN inactivation by mutation seems to also be involved in tumorigenesis, since it occurs in about $90 \%$ of type I carcinomas [11]. Currently, there are no data to suggest that the prognosis for women with HNPCC-associated endometrial cancers is either better or worse than for women with sporadic cancers $[16,24]$. In one study, endometrial carcinoma in HNPCC kindreds was a cause of death in $12 \%$ of cases; in $61 \%$ of cases these patients had a second primary malignancy; and 15\% of cases had more than 2 additional primary cancers. Nieminen et al. [26] studied serial endometrial biopsy samples taken during a 10-year followup of HPNCC mutation carriers and found abnormal MMR protein expression, MSI, or tumor suppressor promotor hypermethylation in various endometrial histologies, including normal and hyperplastic endometria. The most frequently methylated genes were CDH13, RASSF1A, and GSTP1. These defects in MMR and methylation appeared up to 12 years before endometrial carcinoma [26].

PTEN hamartoma tumor syndrome, caused by a germline mutation in PTEN gene on chromosome $10 \mathrm{q}$, comprises a group of disorders including Cowden syndrome, Bannayan-Riley-Ruvalcaba syndrome, Proteus syndrome, Proteus-like syndrome, and autism spectrum disorder with macrocephaly $[27,28]$. Cowden syndrome, also known as multiple hamartoma syndrome, is an autosomal dominant disorder with high risk of breast, thyroid, and endometrium cancer. The incidence of Cowden syndrome remains unclear due to underdiagnosis from variable penetration and subtle clinical findings [29]. Cowden syndrome is characterized by the development of intestinal hamartomas, facial trichilemmomas and mucocutaneous papillomatosis [29, 30], and is rarely identified before adulthood [28]. PTEN mutations in exon 5, coding for the active site and flanking amino acids, is a common site for mutations in patients with Cowden syndrome, and missense mutations are only found in this active area [30]. However, germline PTEN mutation has been detected in approximately $80 \%$ of Cowden syndrome patients [27]. The lifetime risk for endometrial carcinoma in Cowden syndrome is approximately 5-10\%, compared with a $2.5 \%$ lifetime risk of women in the general population [27, 28]. Cowden syndrome and Bannayan-RileyRuvalcaba syndrome have overlapping phenotypic features. 


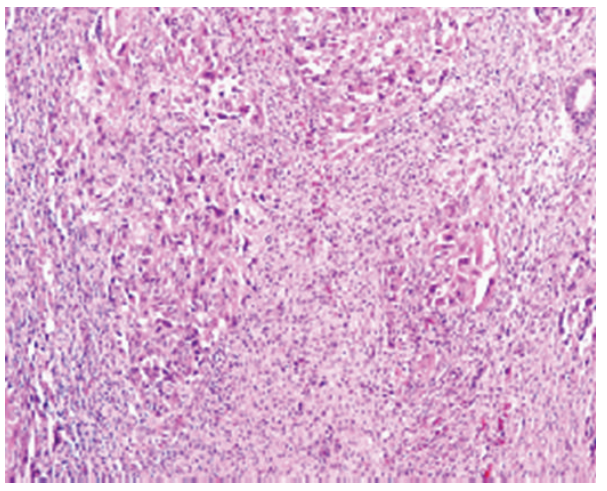

(a)

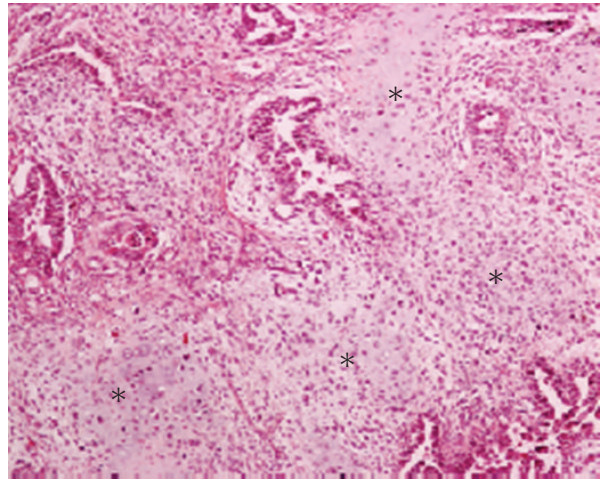

(b)

Figure 4: Carcinosarcoma is composed of two malignant components, carcinomatous and sarcomatous. The epithelial component is usually high grade carcinoma for example, serous/clear cell type. The mesenchymal part comprises either homologous (a), $\times 100$ or heterologous element for example, cartilage or bone. Chondrosarcomatous element $(*)$ is present in $(b), \times 100$.

Bannayan-Riley-Ruvalcaba syndrome is a congenital, autosomal dominant condition manifested by macrocephaly, hamartomatous intestinal polyposis, lipomas, developmental delay or autism or both, and pigmented penile macules $[29,31]$. Unlike Cowden syndrome, Bannayan-RileyRuvalcaba syndrome tends to be diagnosed at early life [28]. Approximately $60 \%$ of Bannayan-Riley-Ruvalcaba syndrome patients have an identifiable germline mutation in PTEN gene [27]. This syndrome also has the same increased risk of cancer as Cowden syndrome [29].

\section{Carcinosarcoma}

Carcinosarcomas (formerly known as malignant mixed mesodermal or mullerian tumors) are highly aggressive, biphasic neoplasms composed of carcinomatous and sarcomatous components (Figure 4). Carcinosarcomas account for $1-2 \%$ of all malignancies of uterine corpus [1] and usually present in postmenopausal women. Uterine bleeding is the most frequent presenting symptom. These tumors have traditionally been regarded as a subtype of uterine sarcomas or as a mixture of true carcinoma and sarcoma, but they are now regarded as metaplastic carcinomas or carcinomas with sarcomatous metaplasia [1, 18, 32-34]. Carcinosarcomas can be classified as type II endometrial carcinomas and their epithelial component can resemble high grade endometrioid, serous or clear cell carcinoma [35]. Etiologically, a few cases may be secondary to prior pelvic irradiation. In addition, an association between long term tamoxifen therapy and development of uterine carcinosarcoma has been suggested [32].

Schipf et al. [36] analyzed a series of 30 paraffinembedded carcinosarcomas, including 24 ovarian and 6 uterine, using fluorescence in situ hybridization (FISH) and CGH. Many carcinosarcomas contained aberrations on chromosome 8 and 20 detected by FISH. FISH showed $C$ MYC (8q24.12) and ZNF217 (20q13.2) amplification in 78\% and $87 \%$, respectively. The results demonstrate a uniform pattern of chromosomal gains and losses in CGH analysis.
Gains or amplifications of $8 \mathrm{q}$ are the most common genetic aberration in carcinosarcomas [35]. One of the genes located within $8 \mathrm{q}$ is $C-M Y C$ (8q24) found to be amplified in 18 of 23 uterine and ovarian carcinosarcomas through FISH and overexpression in 9 of 9 uterine carcinosarcomas through immunostaining. C-MYC amplification is often present in carcinomas but was also present in 6 of 12 uterine leiomyomas and 11 of 23 uterine leiomyosarcomas [35].

LOH was seen in 5 of 6 uterine carcinosarcomas, and identical alleles were lost in the epithelial and mesenchymal components. p53 mutations and LOH for TP53 occur frequently in both carcinosarcoma components which are associated with frequent protein overexpression. Sherman et al. [18] reported immunoreactivity of p53 in 7 (70\%) of 10 carcinosarcomas and noted that the similar staining pattern presented in both carinomatous and sarcomatous areas. In about $20 \%$ of carcinosarcomas, MSI-high was described with an $83 \%$ concordance between the carcinomatous and sarcomatous components [1]. One study found identical mutations of $p 53$ and KRAS in the two components [33]. Fujii et al. [37] analyzed allelic status with polymorphous microsatellite markers on 172 carcinomatous/sarcomatous foci after microdissection of 17 carcinosarcomas. A close relationship between the carcinomatous and the sarcomatous component was found. No difference was seen in CGH patterns of carcinosarcomas [36]. Moreover, there is evidence that in most carcinosarcomas, the carcinomatous and the sarcomatous components are genetically the same, as shown for 21 of 25 carcinosarcomas (84\%) using the human androgen receptor (HUMARA) for detection of Xchromosome inactivation. These results support a monoclonal origin of uterine carcinosarcomas and one can hypothesize that either the sarcomatous component develops from the carcinomatous component (conversion theory) or both are derived from a stem cell that undergoes divergent differentiation (combination theory) [33]. In the process of epithelial-mesenchymal transition, cells of epithelial origin lose epithelial characteristics and polarity acquiring a mesenchymal phenotype with increased migratory behavior. 


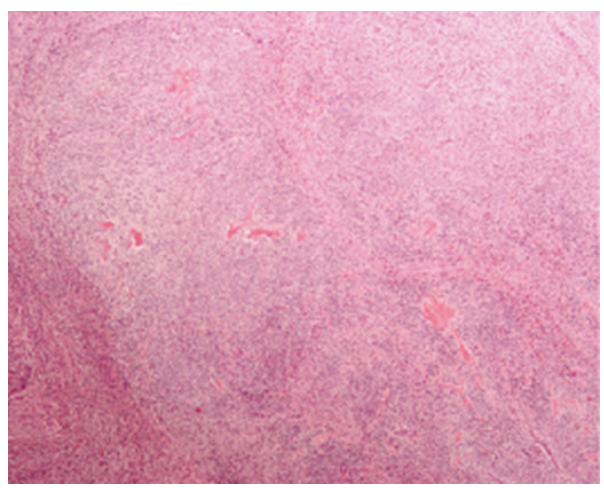

(a)

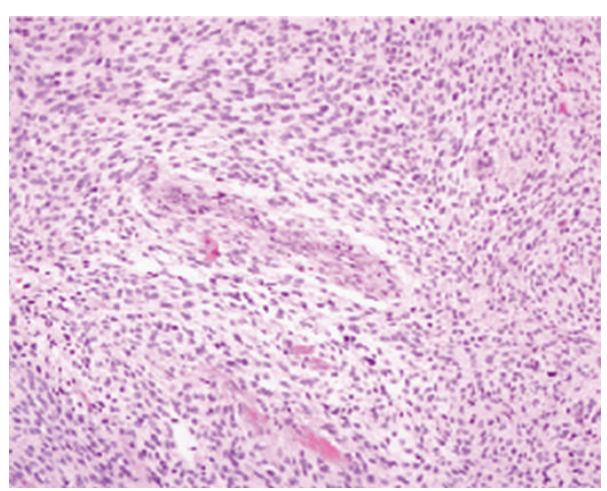

(b)

FIGURE 5: Endometrial stromal sarcoma, low grade is circumscribed from the surrounding myometrium (a), $\times 40$; and higher magnification of endometrial stromal sarcoma shows round uniform tumor cells resembling the stroma of proliferative endometrium with low mitotic rate (b), $\times 200$.

By molecular mechanisms, down-regulation of epithelial markers and up-regulation of mesenchymal markers result in acquisition of a fibroblast-like morphology with cytoskeleton reorganization and increase in motility, invasiveness, and metastatic capacity. A hallmark of epithelial-mesenchymal transition is loss of E-cadherin expression. A number of specific transcription factors, including Snail, Slug, SIP-1, and Twist, contribute to induction of epithelial mesenchymal transition, acting as transcriptional repressors of the Ecadherin gene. The oncogenic serine/threonine kinase AKT also promotes the process, modulating several signaling and transcriptional networks linking $\mathrm{Wnt} / \beta$-catenin, NF- $\kappa \mathrm{B} / \mathrm{p} 65$, and $\mathrm{Rb}[38]$. However, some investigators also found that $\mathrm{a}$ subset of carcinosarcoma was biclonal tumor, consisting of independent unrelated carcinomas and sarcomas, according to $\mathrm{X}$ chromosome inactivation and clinicopathological criteria [35]. In two collision reported tumors, the carcinomatous and sarcomatous components were histologically separate, with no intermingling, and there was a nodal metastasis that consisted purely of the sarcomatous element from one of these tumors [33]. One study examined 26 carcinosarcomas and found adenosarcoma-like components in 4 cases, suggesting that many of the true collision lesions may arise from malignant transformation of either benign epithelium within an adenosarcoma or adjacent benign endometrium [35]. The prognosis of the collision tumor depends on the most aggressive component, and may be better than for a carcinosarcoma of similar stage [33]. Overall, the carcinomatous component has been shown to have a more aggressive behavior and be a better predictor of clinical outcome in carcinosarcomas [35].

\section{Endometrial Stromal Sarcoma and Undifferentiated Endometrial Sarcoma}

Endometrial stromal sarcoma and undifferentiated endometrial sarcoma are in the same neoplastic spectrum. Diagnosis of endometrial stromal tumors has been based on histologic criteria. Low grade endometrial stromal sarcoma is composed of uniform, oval to spindle-shape cells of endometrial stromal-type with numerous small arterioles that resemble the spiral arterioles of late secretory endometrium. Mitotic rate is not a consideration in the distinction between low and high grade stromal sarcoma. In addition, characteristic tongue-like growth of the stromal cells into the myometrium and/or myometrial vasculature is noted [39, 40] (Figure 5). Endometrial stromal sarcoma usually occurs in middle aged women [41], and most present with uterine bleeding. Undifferentiated endometrial sarcoma, on the other hand, is defined as a highgrade neoplasm that lacks specific differentiation and bears no histological resemblance to endometrial stroma. Also, undifferentiated endometrial sarcomas have marked nuclear pleomorphism with high mitotic rate and display destructive myometrial invasion $[40,42]$. Undifferentiated endometrial sarcomas should be diagnosed only after extensive sampling to exclude smooth or skeletal muscle differentiation, to exclude high grade leiomyosarcoma or rhabdomyosarcoma. Carcinosarcoma or adenosarcoma with sarcomatous overgrowth should also be excluded before making the diagnosis of undifferentiated endometrial sarcomas [39, 40].

In endometrial stromal sarcomas, the tumor cells are typically immunoreactive for estrogen and progesterone receptors, CD10, vimentin, and sometimes focally with actin, while they are generally negative for desmin, and h-caldesmon. Expression of androgen receptor is observed in $41 \%$ of examined sarcoma cases [41]. Approximately $70 \%$ of low grade endometrial stromal sarcomas also expresses epidermal growth factor receptor (EGFR; HER1). Undifferentiated endometrial sarcomas are estrogen and progesterone receptor negative, but a high proportion is EGFR positive. Endometrial stromal sarocmas are typically diploid with a low S-phase fraction whereas S-phase fraction exceeds $10 \%$ in undifferentiated endometrial sarcomas [41]. No c-kit (CD117) expression has been demonstrated in endometrial stromal sarcomas [43]. Liegl et al. [43] found 22 of 37 endometrial stromal sarcomas showed platelet-derive growth factor (PDGF) $\alpha$ (CD140 $\alpha)$ and 8 of 37 endometrial stromal sarcomas showed PDGF- $\beta$ expression. 
In contrast to epithelial endometrial carcinoma, endometrial stromal tumors are characterized by distinct cytogenetic abnormalities, particularly translocations leading to gene fusion. Cytogenetic studies reported to-date are primarily for low grade endometrial stromal sarcomas, mostly showing rearrangement of chromosomes 6,7 , and 17 $[44,45]$. Loss of chromosome arm $7 \mathrm{p}$ (55.6\% of the cases) is the most frequent aberration and may play a role in tumor development and progression [41]. Reverse transcription polymerase chain reaction (RT-PCR) and FISH studies on large series showed the presence of $\mathrm{t}(7 ; 17)(\mathrm{p} 15 ; \mathrm{q} 21)$, leading to the fusion of two zinc finger genes, JAZF1 (juxtaposed with another zinc finger gene 1) and JJAZ1 (joined to JAZF1; also named SUZ12, suppressor of zeste-12 protein). JAZF1 is expressed in normal endometrial stroma, but the specific functions of the JAZF1 and the JJAZ1 genes as well as the $J A Z F 1 / J J A Z 1$ fusion gene are still unknown $[1,42]$. Based on the evidence of loss of expression for normal versions of JAZF1 in multiple tumors suggests a possible role of this gene as a tumor suppressor [42]. This gene fusion is a distinctive molecular genetic alteration for endometrial stromal sarcoma and benign endometrial stromal nodules $[1,41]$. The JAZF1/JJAZ1 fusion gene is frequently present in classical endometrial stromal sarcomas and less often in cases with variant histology [46]. However, of seven high-grade endometrial stromal sarcomas/undifferentiated endometrial sarcomas studied, only three cases showed evidence of the fusion [42]. In contrast, many studies reported the fusion gene to be absent in undifferentiated endometrial sarcomas [41]. The fusion gene is not present in normal endometrial stroma [41]. The presence of the JAZF1/JJAZ1 fusion gene within the spectrum of endometrial stromal tumors from benign to malignant raises possibility that the endometrial stromal nodule may transform into malignant endometrial stromal sarcoma [41, 47]. The frequencies of this gene fusion in low grade endometrial stromal sarcoma have been reported in many studies showing a wide range of positivity, $23-80 \%[44,46,48,49]$. The studies with RT-PCR only can give false-positive results due to PCR contamination. FISH may be useful as a complementary technique to exclude the possibility of false positive contamination of cases by RTPCR [44]. Although the JAZF1/JJAZ1 fusion gene seems to be the major molecular alterations in endometrial stromal sarcomas, there is some evidence for alternative pathways in the development of endometrial stromal sarcomas. A major subgroup of endometrial stromal sarcomas has been found to have translocations involving short arm of chromosome 6, particularly band 6p21 [41, 44, 45]. Micci et al. [50] showed that the PHF1 (PHD finger protein 1) gene in 6 p21 was recombined with two different partners, (i) with $J A Z F 1$ gene showing a $6 \mathrm{p} ; 7 \mathrm{p}$ rearrangement, which results in the formation of a JAZF1/PHF1 fusion gene and (ii) with EPC1 (enhancer of polycomb) gene in 10p11 that had a $6 ; 10 ; 10$ translocation. Panagopoulos et al. [45] introduced that a low-grade endometrial stromal sarcoma cell line carrying a $\operatorname{der}(7) \mathrm{t}(6 ; 7)(\mathrm{p} 21 ; \mathrm{p} 22)$ also harbors a JAZF1/PHF1 fusion. Both $\mathrm{t}(7 ; 17)$ and $\mathrm{t}(6 ; 7)$ comprise $62 \%$ of the reported endometrial stromal sarcomas [44]. Additionally, few endometrial stromal sarcoma cases were reported with a $\mathrm{t}(\mathrm{X} ; 17)(\mathrm{p} 11.2 ; \mathrm{q} 23)$ and a $\mathrm{t}(10 ; 17)(\mathrm{q} 22 ; \mathrm{p} 13)$ [51-53]. Although JAZF1/JJAZ1 fusion may not be universally present in all low grade endometrial stromal sarcoma, this aberration may still be diagnostically useful [44]. The JAZF1/JJAZ1 fusion has been identified in areas of smooth muscle differentiation in endometrial stromal neoplasms $(50 \%$ of the cases). This finding supports that the endometrial stromal and smooth muscle components of these tumors have the same origin, either from a common precursor cell with pluripotential differentiation or from endometrial stromal cells that have undergone smooth muscle metaplasia [44, 54]. Halbwedl et al. [55] described 9 cases of low grade endometrial stromal sarcoma and 3 cases of undifferentiated endometrial sarcoma in aCGH study revealing a variety of gains and losses that apparently did not correlate with morphology. There is no accumulation of aberrations in undifferentiated endometrial sarcoma compared to endometrial stromal sarcoma, indicating these two types of uterine sarcomas are probably not related to each other.

$\mathrm{LOH}$ and MSI have been evaluated in both low grade endometrial stromal sarcomas (20 cases) and undifferentiated endometrial sarcomas (3 cases). LOH with at least one polymorphic DNA marker was identified in all 3 cases $(100 \%)$ of undifferentiated endometrial sarcomas, 10 (50\%) low-grade endometrial stromal sarcomas and $2(50 \%)$ benign endometrial stromal nodules. Moreover, concurrent and independent $\mathrm{LOH}$ were noted in adjacent normal appearing myometrium or endometrium, either close to or at a distance from the tumors [41]. LOH was mostly identified at PTEN, a tumor suppressor gene located on chromosome 10q [56]. No tumor was associated with MSI $[41,55]$. Loss of functions of certain tumor suppressor genes such as PTEN in surrounding nontumor uterine tissues could influence and facilitate tumor proliferation, cellular spread, and invasion by malignant endometrial stromal cells [41]. However, one should keep in mind the false positive scoring of LOH in normal tissues may occur both from the imperfect methodology and from contamination by tumor samples/cells. The use of repeated experiments and several polymorphic markers has been advised to overcome these methodology problems [56]. Other frequently altered loci by LOH were at 14q32 (D14S267) and 3p (D3S1300). The former locus is frequently altered in uterine leiomyosarcoma but, in addition, in a variety of epithelial neoplasms such as ovarian, colorectal and esophageal carcinoma. Locus D3S1300 harbors the FHIT gene which is frequently mutated in cervical carcinoma of the uterus. LOH for TP53 and p53 overexpression are rarely present in endometrial stromal sarcomas $(5 \%$ and $15 \%$, resp.). The importance of $p 53$ mutations for the development of undifferentiated endometrial sarcomas is not evident, but p53 overexpression was detected in three of four high-grade stromal sarcomas [1]. Furthermore, Kurihara et al. [49] have recently found frequent nuclear accumulation of p53 and TP53 gene missense mutations in undifferentiated endometrial sarcoma with nuclear pleomorphism, $3(50 \%)$ of 6 cases. There is no evidence of $p 53$ aberration in 18 low grade endometrial stromal sarcomas and 7 cases undifferentiated endometrial sarcoma with nuclear uniformity. p53 alteration may be 
TABLE 3: Typical immunoprofile of type I endometrioid carcinoma and type II serous carcinoma.

\begin{tabular}{lcc}
\hline & $\begin{array}{c}\text { Endometrioid } \\
\text { carcinoma }\end{array}$ & $\begin{array}{c}\text { Serous } \\
\text { carcinoma }\end{array}$ \\
\hline $\begin{array}{l}\text { Estrogen and } \\
\text { progesterone receptors }\end{array}$ & + & - \\
PTEN & - & + \\
$\beta$-catenin & + & - \\
p53 & - & + \\
HER2/neu & - & + \\
\hline
\end{tabular}

+ : positive result, - : negative result

one different pathway that contributes the tumorigenesis of undifferentiated endometrial sarcoma. Expression of SFRP4 and $\beta$-catenin is also detected. SFRP4 acts in Wnt-signaling pathway, which is a complex cascade of heterogeneous molecules playing an important role in organ development, via $\beta$-catenin. SFRP4 is expressed in normal endometrial stromal cells but not in glandular epithelium. Compared with normal endometrium, the expression of SFRP4 was decreased in both low grade endometrial stromal sarcomas and undifferentiated endometrial sarcomas. Through its involvement in the Wnt signaling pathway, SFRP4 may act as a tumor suppressor by regulating the cytosolic $\beta$-catenin pool in the cell. Beta-catenin regulates in the opposite manner to SFRP4, being particularly increased in undifferentiated sarcoma [57]. Dysregulation of these pathways allows $\beta$ catenin to accumulate and translocate to the nucleus, where it forms complexes with T-cell factor/lymphoid enhancing factor (TCF/LEF) leading to uncontrolled cell growth and carcinogenesis [57]. High level nuclear staining for $\beta$-catenin was seen in $40 \%$ of endometrial stromal sarcomas and may be used as a diagnostic tool [42].

\section{Diagnostic Utility Based on the Molecular Knowledge}

7.1. Endometrioid Carcinoma versus Serous/Clear Cell Carcinoma. At times, the histological type of endometrial carcinoma is not clearly defined, especially in poorly differentiated tumors, and knowledge of the dualistic model, with the common molecular changes in each type, can help clarify the diagnosis. If there is non-carcinomatous endometrium present, the presence of hyperplasia is supportive evidence of an endometrioid carcinoma, whereas atrophic endometrium is supportive of non-endometrioid carcinoma.

Molecular studies on endometrium are not often performed in most hospital surgical pathology laboratories today; however, immunohistochemical studies can detect the abnormal protein products of the gene mutations. Therefore, we can exploit our knowledge of the dualistic model and their typical gene mutations and use the immunoprofile as a diagnostic tool, in concert with the histomorphologic features to specify the tumor type, particularly in difficult cases such as in the differentiation between high-grade endometrioid carcinoma and serous carcinoma (Table 3 ).
7.2. Endometrial Stromal Sarcoma versus Undifferentiated Sarcoma. The distinct molecular alteration described in the majority of endometrial stromal sarcomas is the $\mathrm{t}(7 ; 17)(\mathrm{p} 15 ; \mathrm{q} 21)$ leading to the formation of fusion gene JAZF1/JJAZ1, which can be detected by RT-PCR or FISH assays. Thus, in the problematic cases in which the differentiatial diagnosis is between endometrial stromal sarcoma and undifferentiated sarcoma, we look for the fusion gene to make this distinction.

7.3. Uterine Smooth Muscle Neoplasm versus Endometrial Stromal Tumors. Uterine smooth muscle neoplasm is defined as a mesenchymal tumor composed of cells with smooth muscle differentiation, particularly highly cellular leiomyomas may have morphologically overlapped features of endometrial stromal tumors. According to histologic criteria for differential, immunostainings may help to correct the final diagnosis, particularly in difficult cases. Neoplastic endometrial stromal cells typically express vimentin, musclespecific and smooth muscle actin and may be positive for desmin. In addition, CD10, initially thought to be a specific marker for endometrial stromal tumors, can be demonstrated in uterine smooth muscle tumors, commonly in highly cellular leiomyomas and leiomyosarcomas. Other antibodies that give positive staining in smooth muscle tumors useful in this differential diagnosis includes $\mathrm{h}$ caldesmon, histone deacetylase 8 (HDAC8), smooth muscle myosin and oxytocin receptor [39]. However, none of these markers can completely specify the smooth muscle/endometrial stroma lineage of the tumor, a panel of the antibodies should be used [39].

The molecular alterations in smooth muscle tumor are complex, especially in leiomyosarcomas. The translocation $\mathrm{t}(12 ; 14)$ (q15; $\mathrm{q} 23-24)$ has been noted in a high proportion of leiomyomas $[41,58]$. By $\mathrm{CGH}$, leiomyosarcomas have the most frequent losses including $10 \mathrm{q}, 11 \mathrm{q}, 13 \mathrm{q}$, and $2 \mathrm{p}$ while the most common gains are $X p, 1 q, 5 p, 8 q, 12 q, 17 p$ and $19 p[41,59,60]$. There are a variety of genetic changes and mutations inclusive of TP53 and MDM2 expression associated with progression of leiomyosarcomas [41]. $\mathrm{LOH}$ of $10 \mathrm{q}$ is found in more than half of leiomyosarcomas [41]. Leiomyosarcomas exhibit a significantly higher frequency of allelic loss (52\%) compared with benign leiomyomas (18\%) and smooth muscle tumors of uncertain malignant potential (21\%) [41].

\section{Therapeutic Considerations: Molecular Targeted Therapy}

Development of targeted anticancer drugs is the direct result of knowledge of the molecular profile of endometrial neoplasms. Drug targets may focus on genes that affect apoptosis, signal transduction, epigenetic modification, drug resistance, protein folding and degradation, cell cycle progression, hormone receptor activity, and angiogenesis [4]. The drugs that comprise targeted therapy include small molecular weight inhibitors, monoclonal antibodies, antisense and gene therapy [61]. At this time, essentially only 
endometrial carcinomas have been tested with targeted therapy. Carcinosarcomas and endometrial stromal sarcomas are relatively uncommon neoplasms, and there has little experience with specific therapies for these tumors, though there is definitely future potential.

8.1. mTOR Inhibitors. The phosphatidylinositol-3-kinase (PI3K)-serine/threonine kinase (AKT)-mammalian target of the rapamycin (mTOR) signaling pathway plays a central role in the regulation of cell growth, proliferation, and apoptosis. In in vitro studies, cells with PTEN inactivation in endometrioid carcinoma are sensitive to mTOR inhibitors, since the loss of PTEN leads to constitutive activation of downstream components, which in turn up-regulates mTOR activity [62]. Potential therapies targeting the mTOR pathway include the mTOR inhibitors temsirolimus (CCI779), everolimus (RAD001), and deforolimus (AP23573) [4]. In a phase II study of temsirolimus activity in patients with advanced or recurrent endometrial cancer, 5 of $19(26 \%)$ evaluable patients had a partial response and $12(63 \%)$ had stable disease $[62,63]$. In addition to mTOR inhibitors, other agents targeting components of the mTOR-AKTPI3K-PTEN pathway have also been developed, including enzastaurin (a PI3K inhibitor) and triciribine (an AKT inhibitor) [4].

8.2. EGFR Inhibitors/Anti-HER2/neu. Epidermal growth factor receptor (EGFR) family members (ERBB1 (EGFR or HER1), ERBB2 (HER2/neu), ERBB3 (HER3), ERBB4 (HER4)) are tyrosine kinase receptors that are activated by binding to epidermal growth factor (EGF)-like growth factor, leading to downstream phosphorylation or dephosphorylation of signaling molecules that involved in cell cycle and apoptosis [63]. Sixty to $80 \%$ of endometrial carcinomas overexpress EGFR [4]. In addition, EGFR expression has been described in approximately $70 \%$ of endometrial stromal sarcomas [41]. EGFR overexpression has been reported in high grade carcinomas with deep myometrial invasion, positive peritoneal washings and poor survival [61, 63]. The anti-EGFR agents result in down regulation of the MAPK and PI3K/AKT signaling pathways. However, the anti-tumor activity has been described in a minority of the patients treated. Antagonists to EGFR include small molecule tyrosine kinase inhibitors (gefitinib, erlotinib, and lapatinib) and the anti-EGFR monoclonal antibody cetuximab [62]. Experimental observation data have been shown that EGFR inhibitors could be more effective in endometrioid endometrial carcinoma than in uterine papillary serous carcinoma [63]

As described above, HER2/neu gene overexpression and amplification have been found in up to $80 \%$ of nonendometrioid endometrial carcinoma, and in 10$30 \%$ of endometrioid endometrial carcinoma. The usage of trastuzumab, a monoclonal antibody directed against HER2/neu, has been tested in endometrial carcinomas. Villella's group found 5 out of 19 (26\%) patients with papillary serous carcinoma showed HER2/neu overexpression. One of 5 positive HER2/neu patients with advanced disease treated with trastuzumab achieved a complete response and a second patient's disease stabilized [63]. However in another study, Gynecologic Oncology Group (GOG)0181-B, investigated trastuzumab in advanced, recurrent, or persistent endometrial cancer, and its preliminary results showed minimal activity, even in cancers with high overexpression of HER2/neu [4]. Several other monoclonal antibodies targeting members of the ERBB/HER family, including pertuzumab, cetuximab, and panitumumab, are currently being investigated [4].

8.3. Antiangiogenics. Vascular endothelial growth factors (VEGF) expression has been found in $56-100 \%$ of endometrial carcinomas [63] and has been correlated with high histologic grade, deep myometrial invasion, angiolymphatic invasion, nodal metastasis, and short disease-free survival $[63,64]$. VEGF, particularly VEGF-A, plays a key role in angiogenesis and increased permeability of tumor-associated blood vessels. Monoclonal antibodies targeting VEGF, bevacizumab and sorafenib, have been developed. Kamat and coworkers [64] injected Ishikawa cell line into uterine horn of nude mice in one group and Hec-1A cell lines in the other group and treated the mice with docetaxel and/or bevacizumab. The combination of both agents had a greater efficacy in tumor growth inhibition than a single agent. Currently, GOG-229-E is being studied in a phase II trial of single agent bevacizumab in patients with recurrent endometrial carcinoma [64].

\section{Conclusion}

Knowledge of the molecular profiles of endometrial neoplasms assists in the diagnosis, prognosis, and treatment of endometrial neoplasms. Endometrial carcinoma can be broadly divided into two categories based on clinical behavior and morphologic phenotype, with good correlation to the molecular findings. Type I endometrial carcinoma represents an estrogen-related tumor, which usually arises in the setting of endometrial hyperplasia and have good prognosis. They are associated with a number of welldescribed genetic alterations including mutations of PTEN, KRAS, $\beta$-catenin, PIK3CA, and inactivation of DNA mismatch repair. Targets for molecular therapy in endometrial carcinoma include agents that inhibit components of the AKT-PI3K-PTEN pathway. Type II endometrial cancers are not estrogen-related and have poor prognosis. Mutations of p53 are present in approximately 90\% of this tumor type. Carcinosarcoma is considered to be a high-grade carcinoma with sarcomatous differentiation and a high frequency of C-MYC mutations and $\mathrm{LOH}$ of $p 53$. The majority of endometrial stromal nodules and stromal sarcomas seem to originate from the abnormal JAZF1/JJAZ1 gene fusion. The molecular biology of undifferentiated endometrial sarcomas is still not clearly delineated. In the near future; additional molecular studies should further elucidate the unclear pathogenesis and provide new targets for diagnosis and treatment. 


\section{References}

[1] S. F. Lax, "Molecular genetic changes in epithelial, stromal and mixed neoplasms of the endometrium," Pathology, vol. 39, no. 1, pp. 46-54, 2007.

[2] S. G. Silverberg, R. J. Kurman, F. Nogales, G. Mutter, R. A. Kubik-Huch, and F. A. Tavassoli, "Epithelial tumors and related lesions," in Pathology and Genetics of Tumors of the Breast and Female Organs, P. Devilee and F. A. Tavassoli, Eds., IARC Press, Lyon, France, 2003.

[3] M. J. Horner, L. A. G. Ries, M. Krapcho, et al., "SEER Cancer Statistics Review 1975-2006," 2009, http://seer.cancer.gov/csr/ 1975_2006/.

[4] N. Bansal, V. Yendluri, and R. M. Wenham, "The molecular biology of endometrial cancers and the implications for pathogenesis, classification, and targeted therapies," Cancer Control, vol. 16, no. 1, pp. 8-13, 2009.

[5] J. V. Bokhman, "Two pathogenetic types of endometrial carcinoma," Gynecologic Oncology, vol. 15, no. 1, pp. 10-17, 1983.

[6] A. Di Cristofano and L. H. Ellenson, "Endometrial carcinoma," Annual Review of Pathology, vol. 2, pp. 57-85, 2007.

[7] D. Llobet, J. Pallares, A. Yeramian, et al., "Molecular pathology of endometrial carcinoma: practical aspects from the diagnostic and therapeutic viewpoints," Journal of Clinical Pathology, vol. 62, no. 9, pp. 777-785, 2009.

[8] X. Matias-guiu, L. Catasus, E. Bussaglia, et al., "Molecular pathology of endometrial hyperplasia and carcinoma," Human Pathology, vol. 32, no. 6, pp. 569-577, 2001.

[9] J. Prat, A. Gallardo, M. Cuatrecasas, and L. Catasús, "Endometrial carcinoma: pathology and genetics," Pathology, vol. 39, no. 1, pp. 72-87, 2007.

[10] F.-S. Liu, "Molecular carcinogenesis of endometrial cancer," Taiwanese Journal of Obstetrics and Gynecology, vol. 46, no. 1, pp. 26-32, 2007.

[11] S. F. Lax, "Molecular genetic pathways in various types of endometrial carcinoma: from a phenotypical to a molecularbased classification," Virchows Archiv, vol. 444, no. 3, pp. 213 223, 2004.

[12] J. L. Hecht and G. L. Mutter, "Molecular and pathologic aspects of endometrial carcinogenesis," Journal of Clinical Oncology, vol. 24, no. 29, pp. 4783-4791, 2006.

[13] A. Doll, M. Abal, M. Rigau, et al., "Novel molecular profiles of endometrial cancer-new light through old windows," Journal of Steroid Biochemistry and Molecular Biology, vol. 108, no. 3-5, pp. 221-229, 2008.

[14] S. N. Westin, R. R. Broaddus, L. Deng, et al., "Molecular clustering of endometrial carcinoma based on estrogeninduced gene expression," Cancer Biology \& Therapy, vol. 8, no. 22, pp. 2126-2135, 2009.

[15] L. Catasus, A. Gallardo, M. Cuatrecasas, and J. Prat, "Concomitant PI3K-AKT and p53 alterations in endometrial carcinomas are associated with poor prognosis," Modern Pathology, vol. 22, no. 4, pp. 522-529, 2009.

[16] Y. Karamurzin and J. K. L. Rutgers, "DNA mismatch repair deficiency in endometrial carcinoma," International Journal of Gynecological Pathology, vol. 28, no. 3, pp. 239-255, 2009.

[17] H. Tashiro, M. S. Blazes, R. Wu, et al., "Mutations in PTEN are frequent in endometrial carcinoma but rare in other common gynecological malignancies," Cancer Research, vol. 57, no. 18, pp. 3935-3940, 1997.
[18] M. E. Sherman, M. E. Bur, and R. J. Kurman, "p53 in endometrial cancer and its putative precursors: evidence for diverse pathways of tumorigenesis," Human Pathology, vol. 26, no. 11, pp. 1268-1274, 1995.

[19] J. Pallares, E. Bussaglia, J. L. Martínez-Guitarte, et al., "Immunohistochemical analysis of PTEN in endometrial carcinoma: a tissue microarray study with a comparison of four commercial antibodies in correlation with molecular abnormalities," Modern Pathology, vol. 18, no. 5, pp. 719-727, 2005.

[20] A. Velasco, E. Bussaglia, J. Pallares, et al., "PIK3CA gene mutations in endometrial carcinoma. Correlation with PTEN and K-RAS alterations," Human Pathology, vol. 37, no. 11, pp. 1465-1472, 2006.

[21] A. Dutt, H. B. Salvesen, H. Greulich, W. R. Sellers, R. Beroukhim, and M. Meyerson, "Somatic mutations are present in all members of the AKT family in endometrial carcinoma," British Journal of Cancer, vol. 101, no. 7, pp. 12181219, 2009.

[22] H. Tashiro, C. Isacson, R. Levine, R. J. Kurman, K. R. Cho, and L. Hedrick, "p53 gene mutations are common in uterine serous carcinoma and occur early in their pathogenesis," American Journal of Pathology, vol. 150, no. 1, pp. 177-185, 1997.

[23] R. A. Ambros, M. E. Sherman, C. M. Zahn, P. Bitterman, and R. J. Kurman, "Endometrial intraepithelial carcinoma: a distinctive lesion specifically associated with tumors displaying serous differentiation," Human Pathology, vol. 26, no. 11, pp. 1260-1267, 1995.

[24] L. A. Meyer, R. R. Broaddus, and K. H. Lu, "Endometrial cancer and lynch syndrome: clinical and pathologic considerations," Cancer Control, vol. 16, no. 1, pp. 14-22, 2009.

[25] K. Garg and R. A. Soslow, "Lynch syndrome (hereditary nonpolyposis colorectal cancer) and endometrial carcinoma," Journal of Clinical Pathology, vol. 62, no. 8, pp. 679-684, 2009.

[26] T. T. Nieminen, A. Gylling, W. M. Abdel-Rahman, et al., "Molecular analysis of endometrial tumorigenesis: importance of complex hyperplasia regardless of atypia," Clinical Cancer Research, vol. 15, no. 18, pp. 5772-5783, 2009.

[27] R. Pilarski, "Cowden syndrome: a critical review of the clinical literature," Journal of Genetic Counseling, vol. 18, no. 1, pp. 1327, 2009.

[28] J. A. Hobert and C. Eng, "PTEN hamartoma tumor syndrome: an overview," Genetics in Medicine, vol. 11, no. 10, pp. 687694, 2009.

[29] K. M. Schmeler, M. S. Daniels, A. C. Brandt, and K. H. Lu, "Endometrial cancer in an adolescent: a possible manifestation of Cowden syndrome," Obstetrics and Gynecology, vol. 114, no. 2, part 2, pp. 477-479, 2009.

[30] M. R. Nelen, W. C. G. Van Staveren, E. A. J. Peeters, et al., "Germline mutations in the PTEN/MMAC1 gene in patients with Cowden disease," Human Molecular Genetics, vol. 6, no. 8, pp. 1383-1387, 1997.

[31] N. E. Lynch, S. A. Lynch, J. McMenamin, and D. Webb, "Bannayan-Riley-Ruvalcaba syndrome: a cause of extreme macrocephaly and neurodevelopmental delay," Archives of Disease in Childhood, vol. 94, no. 7, pp. 553-554, 2009.

[32] W. G. McCluggage, U. Haller, R. J. Kurman, and R. A. Kubik-Huch, "Mixed epithelial and mesenchymal tumors," in Pathology and Genetics of Tumors of the Breast and Female Organs, P. Deville and F. A. Tavassoli, Eds., IARC Press, Lyon, France, 2003. 
[33] W. G. McCluggage, "Malignant biphasic uterine tumours: carcinosarcomas or metaplastic carcinomas?" Journal of Clinical Pathology, vol. 55, no. 5, pp. 321-325, 2002.

[34] W. G. McCluggage, "Uterine carcinosarcomas (malignant mixed Mullerian tumors) are metaplastic carcinomas," International Journal of Gynecological Cancer, vol. 12, no. 6, pp. 687-690, 2002.

[35] L. E. Kernochan and R. L. Garcia, "Carcinosarcomas (malignant mixed müllerian tumor) of the uterus: advances in elucidation of biologic and clinical characteristics," Journal of the National Comprehensive Cancer Network, vol. 7, no. 5, pp. 550-557, 2009.

[36] A. Schipf, D. Mayr, T. Kirchner, and J. Diebold, "Molecular genetic aberrations of ovarian and uterine carcinosarcomasa CGH and FISH study," Virchows Archiv, vol. 452, no. 3, pp. 259-268, 2008.

[37] H. Fujii, M. Yoshida, Z. X. Gong, et al., "Frequent genetic heterogeneity in the clonal evolution of gynecological carcinosarcoma and its influence on phenotypic diversity," Cancer Research, vol. 60, no. 1, pp. 114-120, 2000.

[38] M. Saegusa, M. Hashimura, T. Kuwata, and I. Okayasu, "Requirement of the Akt/ $\beta$-catenin pathway for uterine carcinosarcoma genesis, modulating E-cadherin expression through the transactivation of slug," American Journal of Pathology, vol. 174, no. 6, pp. 2107-2115, 2009.

[39] P. Baker and E. Oliva, "Endometrial stromal tumours of the uterus: a practical approach using conventional morphology and ancillary techniques," Journal of Clinical Pathology, vol. 60, no. 3, pp. 235-243, 2007.

[40] M. R. Hendrickson, F. A. Tavassoli, and R. L. Kempson, "Mesenchymal tumors and related lesion," in Pathology and Genetics of Tumors of the Breast and Female Organs, P. Devilee and F. A. Tavassoli, Eds., IARC Press, Lyon, France, 2003.

[41] F. Moinfar, M. Azodi, and F. A. Tavassoli, "Uterine sarcomas," Pathology, vol. 39, no. 1, pp. 55-71, 2007.

[42] A. A. Sandberg, "The cytogenetics and molecular biology of endometrial stromal sarcoma," Cytogenetic and Genome Research, vol. 118, no. 2-4, pp. 182-189, 2007.

[43] B. Liegl, O. Reich, F. F. Nogales, and S. Regauer, "PDGF- $\alpha$ and PDGF- $\beta$ are expressed in endometrial stromal sarcoma: a potential therapeutic target for tyrosine kinase inhibitors?" Histopathology, vol. 49, no. 5, pp. 545-546, 2006.

[44] M. R. Nucci, D. Harburger, J. Koontz, P. D. Cin, and J. Sklar, "Molecular analysis of the JAZF1-JJAZ1 gene fusion by RTPCR and fluorescence in situ hybridization in endometrial stromal neoplasms," American Journal of Surgical Pathology, vol. 31, no. 1, pp. 65-70, 2007.

[45] I. Panagopoulos, F. Mertens, and C. A. Griffin, "An endometrial stromal sarcoma cell line with the JAZF1/PHF1 chimera," Cancer Genetics and Cytogenetics, vol. 185, no. 2, pp. 74-77, 2008.

[46] H.-Y. Huang, M. Ladanyi, and R. A. Soslow, "Molecular detection of JAZF1-JJAZ1 gene fusion in endometrial stromal neoplasms with classic and variant histology: evidence for genetic heterogeneity," American Journal of Surgical Pathology, vol. 28, no. 2, pp. 224-232, 2004.

[47] F. Moinfar, M.-L. Kremser, Y. G. Man, K. Zatloukal, F. A. Tavassoli, and H. Denk, "Allelic imbalances in endometrial stromal neoplasms: frequent genetic alterations in the nontumorous normal-appearing endometrial and myometrial tissues," Gynecologic Oncology, vol. 95, no. 3, pp. 662-671, 2004.
[48] A. Hrzenjak, F. Moinfar, F. A. Tavassoli, et al., "JAZF1/JJAZ1 gene fusion in endometrial stromal sarcomas. Molecular analysis by reverse transcriptase-polymerase chain reaction optimized for paraffin-embedded tissue," Journal of Molecular Diagnostics, vol. 7, no. 3, pp. 388-395, 2005.

[49] S. Kurihara, Y. Oda, Y. Ohishi, et al., "Endometrial stromal sarcomas and related high-grade sarcomas: immunohistochemical and molecular genetic study of 31 cases," American Journal of Surgical Pathology, vol. 32, no. 8, pp. 1228-1238, 2008.

[50] F. Micci, I. Panagopoulos, B. Bjerkehagen, and S. Heim, "Consistent rearrangement of chromosomal band 6p21 with generation of fusion genes JAZF1/PHF1 and EPC1/PHF1 in endometrial stromal sarcoma," Cancer Research, vol. 66, no. 1, pp. 107-112, 2006.

[51] F. Amant, P. Moerman, I. Cadron, A. Hagemeijer, I. Vergote, and M. Debiec-Rychter, "Endometrial stromal sarcoma with a sole $t(X ; 17)$ chromosome change: report of a case and review of the literature," Gynecologic Oncology, vol. 88, no. 3, pp. 459$462,2003$.

[52] K. Leunen, F. Amant, M. Debiec-Rychter, et al., "Endometrial stromal sarcoma presenting as postpartum haemorrhage: report of a case with a sole $\mathrm{t}(10 ; 17)(\mathrm{q} 22 ; \mathrm{p} 13)$ translocation," Gynecologic Oncology, vol. 91, no. 1, pp. 265-271, 2003.

[53] S. Regauer, W. Emberger, O. Reich, and R. Pfragner, "Cytogenetic analyses of two new cases of endometrial stromal sarcoma-non-random reciprocal translocation $\mathrm{t}(10 ; 17)(\mathrm{q} 22 ; \mathrm{p} 13)$ correlates with fibrous ESS," Histopathology, vol. 52, no. 6, pp. 780-783, 2008.

[54] E. Oliva, L. De Leval, R. A. Soslow, and C. Herens, "High frequency of JAZF1-JJAZ1 gene fusion in endometrial stromal tumors with smooth muscle differentiation by interphase FISH detection," American Journal of Surgical Pathology, vol. 31, no. 8, pp. 1277-1284, 2007.

[55] I. Halbwedl, R. Ullmann, M.-L. Kremser, et al., "Chromosomal alterations in low-grade endometrial stromal sarcoma and undifferentiated endometrial sarcoma as detected by comparative genomic hybridization," Gynecologic Oncology, vol. 97, no. 2, pp. 582-587, 2005.

[56] F. Micci and S. Heim, "Pathogenetic mechanisms in endometrial stromal sarcoma," Cytogenetic and Genome Research, vol. 118, no. 2-4, pp. 190-195, 2007.

[57] A. Hrzenjak, M. Tippl, M.-L. Kremser, et al., "Inverse correlation of secreted frizzled-related protein 4 and $\beta$ catenin expression in endometrial stromal sarcomas," Journal of Pathology, vol. 204, no. 1, pp. 19-27, 2004.

[58] J. C. Hodge, B. J. Quade, M. A. Rubin, E. A. Stewart, P. D. Cin, and C. C. Morton, "Molecular and cytogenetic characterization of plexiform leiomyomata provide further evidence for genetic heterogeneity underlying uterine fibroids," American Journal of Pathology, vol. 172, no. 5, pp. 1403-1410, 2008.

[59] B. Levy, T. Mukherjee, and K. Hirschhorn, "Molecular cytogenetic analysis of uterine leiomyoma and leiomyosarcoma by comparative genomic hybridization," Cancer Genetics and Cytogenetics, vol. 121, no. 1, pp. 1-8, 2000.

[60] J. Yang, X. Du, K. Chen, et al., "Genetic aberrations in soft tissue leiomyosarcoma," Cancer Letters, vol. 275, no. 1, pp. 18, 2009.

[61] A. N. Y. Cheung, "Molecular targets in gynaecological cancers," Pathology, vol. 39, no. 1, pp. 26-45, 2007.

[62] S. M. Temkin and G. Fleming, "Current treatment of metastatic endometrial cancer," Cancer Control, vol. 16, no. 1, pp. 38-45, 2009. 
[63] A. Gadducci, R. Tana, S. Cosio, A. Fanucchi, and A. R. Genazzani, "Molecular target therapies in endometrial cancer: from the basic research to the clinic," Gynecological Endocrinology, vol. 24, no. 5, pp. 239-249, 2008.

[64] A. A. Kamat, W. M. Merritt, D. Coffey, et al., "Clinical and biological significance of vascular endothelial growth factor in endometrial cancer," Clinical Cancer Research, vol. 13, no. 24, pp. 7487-7495, 2007. 


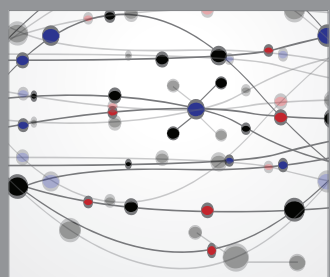

The Scientific World Journal
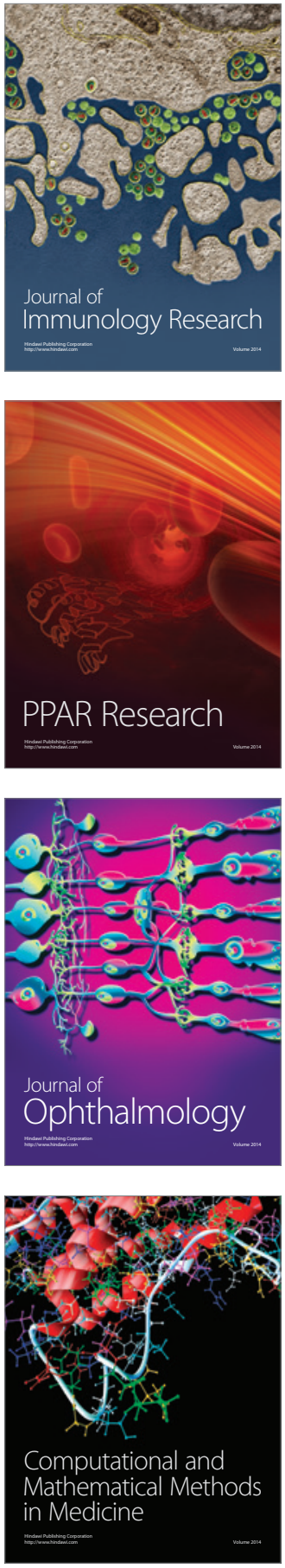

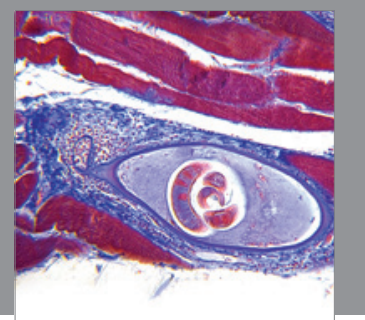

Gastroenterology

Research and Practice
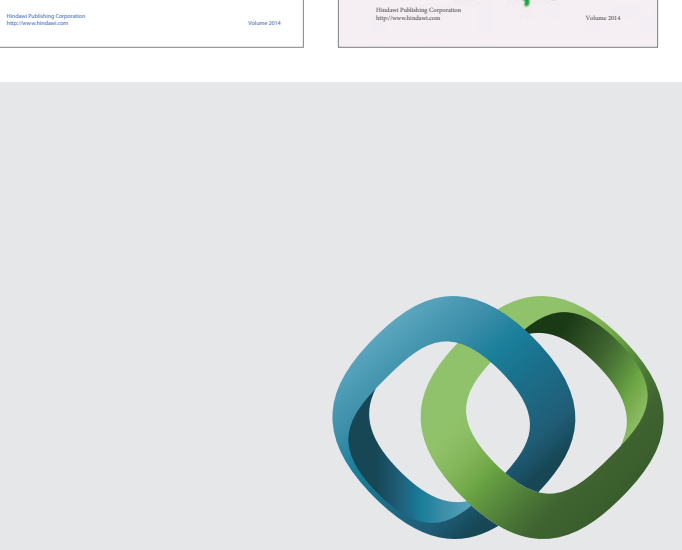

\section{Hindawi}

Submit your manuscripts at

http://www.hindawi.com
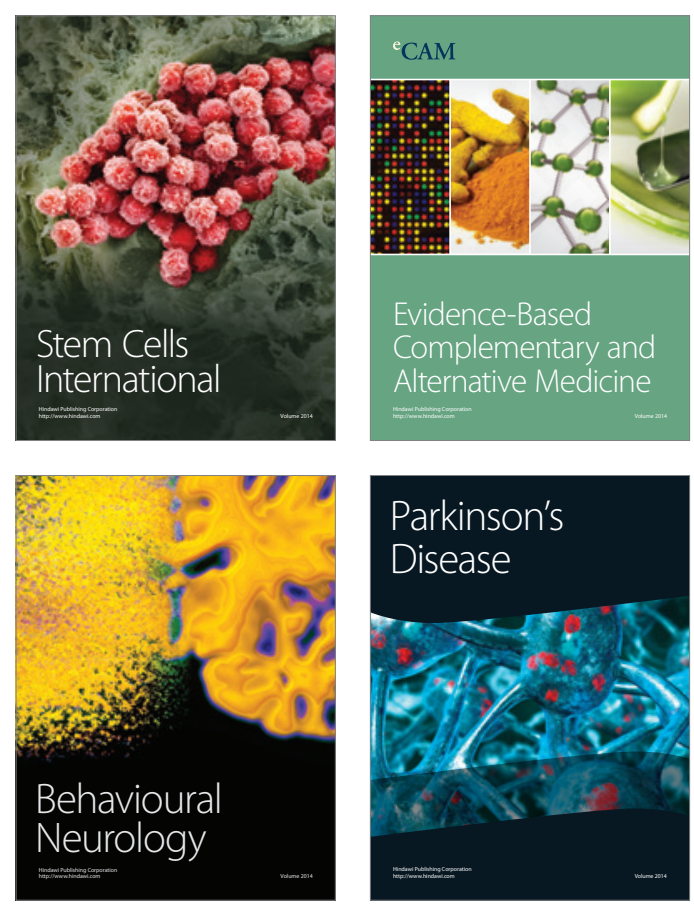

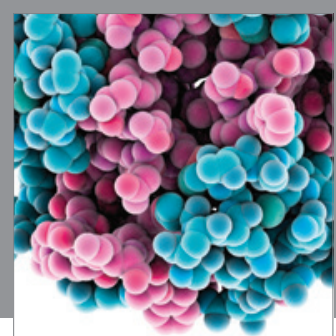

Journal of
Diabetes Research

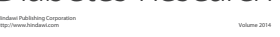

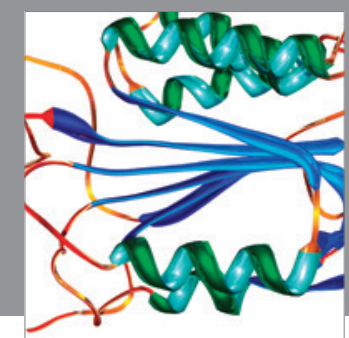

Disease Markers
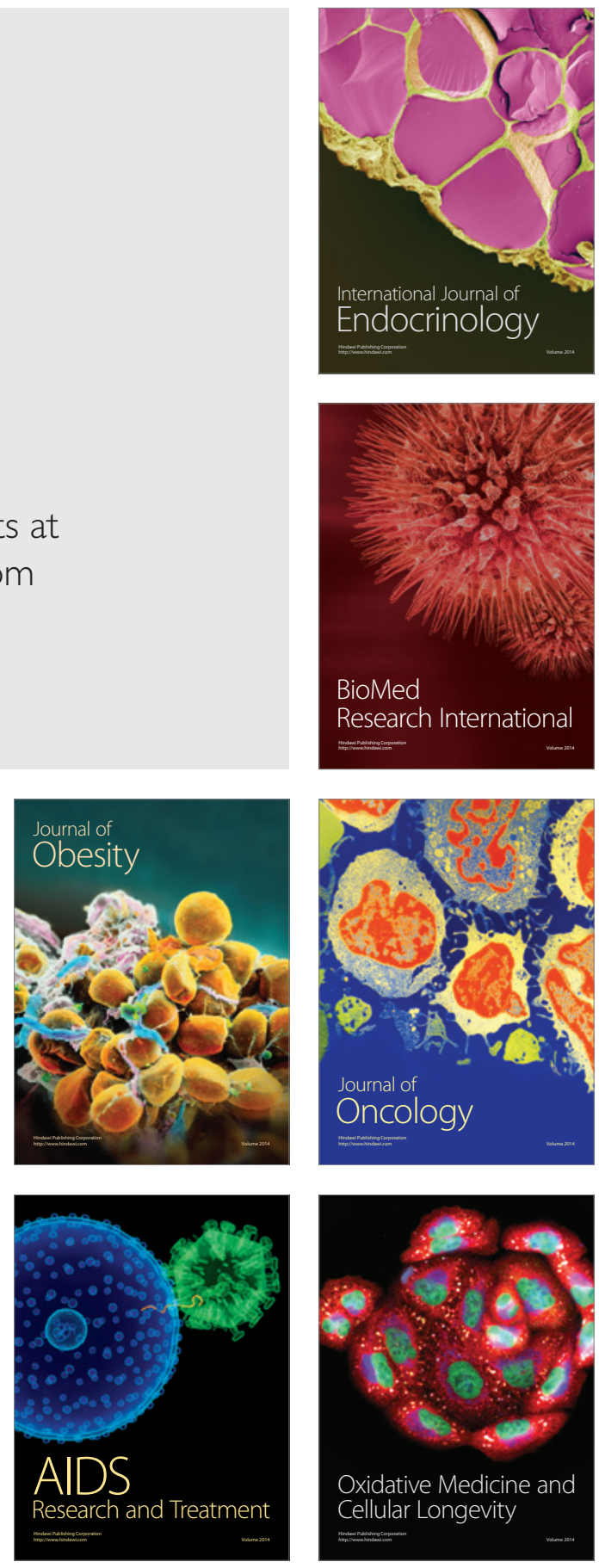